\title{
Deuteration as a Tool for Enhancing the Half-Life of Drug
}

\author{
Vijay Kumar, Archana Dhyani, N Singh
}

\begin{abstract}
The aim of the article is that deuteration of any compounds leads to the enhancement of metabolic activity.The substitution of Carbon-Hydrogen bond by Carbon-Deutrieum help for enhancing pharmacokinetic profile of the drug. Since $C$ $D$ bond is ten time more tough to $C-H$ bond .Nowadays, many drug molecules are deuterated to increase the residence time of the drug as well as diminution the metabolism of the drug.Deuterated drugs also finds various therapeutic applications.The deuterated drugs is also approved by Food and Drug Administration. The deuteration helps in increasing the dwell time of the drug and reducing frequency of dosing.
\end{abstract}

Keywords: Deuteration, half- life, pharmacokinetics, therapeutic effects

\section{INTRODUCTION}

Drug research and development is a more difficult process because of increased cost and risk associated with the process.(1)Deuterium is a isotope of water , containing $0.015 \%$ deuteriumoxide. In nuclear reactors it is basically used as moderators.(2)When living systems are exposed to $\mathrm{D}_{2} \mathrm{O}$ the two affects are basically arises.One is due to effect of $\mathrm{D}_{2} \mathrm{O}$ itself and the second affect is due to capability to substitute hydrogen withdeuterium in body. The carbon -deuteriumbond resistant to enzymatic cleavage as compared to $\mathrm{C}-\mathrm{H}$ bond. The compounds containing $\mathrm{C}-\mathrm{D}$ bonds are more stable than compounds containing $\mathrm{C}-\mathrm{H}$ bond.(3)

$\mathrm{D}_{2} \mathrm{O}$ also has numerous therapeutic applications and it is used as therapeutic agent against pancreatic cancer, stability of cells and tissues, stability of macromolecules and helps in determination of total body water.(4) Recently,a number of patents are granted or filed regarding the potential therapeutic applications of $\mathrm{D}_{2} \mathrm{O} .(5)$

\section{THERAPEUTIC APPLICATION OF DEUTERATED COMPOUNDS}

The deuterateddrug can be used in the treatment of various human diseases.Edward M. Russak et al (2018) studied that first deuterated drugisDeutetrabenazine which is deutrated form of tetrabenazine. Deutetrabenazine is the drug which is accepted by Food and Drug Administration (FDA)and the drug is used in the treatment of Huntington's disease and tardive dyskinesia. (6)

Revised Manuscript Received on November 25, 2019.

Vijay Kumar, Department of Physics, Graphic Era Hill University, Clement Town, Dehradun,Uttarakhand, India

Archana Dhyani, School of Pharmacy, Graphic Era Hill University, Clement Town, Dehradun, Uttarakhand, India.

N Singh, School of Pharmacy, Graphic Era Hill University, Clement Town, Dehradun, Uttarakhand, India.
The deutrated drug is also responsible for improved metabolism of drug. The another study which was done by Graham S Timmins et al (2014) who studied that transformation of drugs into deuterated formhas better pharmacokinetic or toxicological properties. This is because due to the presence stronger deuterium-carbon bond which is responsible for their improved metabolism. The ability of deutriuem oxide for treatment of various diseases ,use of deuteriuem oxide in experiments, artificial synthesis of deuterium-labeled compounds was studied by Wendell Costa Bila (2017).(7)

Deuterated Efavirenz finds use for nephrotoxicity. Scott L. Harbeson et al (2014) studied thatthe deuteratedefavirenzuse to clarify the nephrotoxicity in mice. Efavirenz is ainhibitor of reverse transcriptase which is used for treatment of HIV. The substitution of hydrogenof cyclopropylmethineby means of deuterium, thus dropping the metabolism and reduces the severity of nephrotoxicity.(8)

The secondary amines can be useful in synthesis of larger polymers and organic molecules.The study onoptoelectronic devices was doneby Anwen M. KrauseHeuer 2014. The production of organic molecules or polymers can be done by usingdeuterated arylamines(9).A study was done byRobert B. Raffaet al (2018)in which he studied that the $(\mathrm{C}-\mathrm{H})$ bond has less stability in comparison to $\mathrm{C}-\mathrm{D}$ bond. It was found that if deuterium is located suitably in drug so the carbon deutriuem bond does not undergo metabolic breakdown which leads to enhance the stability of the drug(10).

Similarly, a study was done by Sarah Cargnin (2019) which revealed that there are many deutrated drugs in clinincal development stage. About 20 drugs are under this stage in which 6 drugs reaches the IIIphase clinical trials. Through out previous years more focus is given to the deuterated drugs and in 2018, novel drug, HC-1119, enter in clinical development of deuterated drug.(11).

Another important study was done by Sukhninder Kauret al (2017) where it was emphasize that deuteration take part in enhancing half life of drug. The bond formation between carbon hydrogen is weak as compared to carbon with deuterium. This ultimately leads to increase in the biological half-life of the drug (12). Cuibo Liu (2018) researched the halides deuteration using heavy water. The deuteration strategy show improved results and tolerances. The deuterated acids and alkynes are useful in various reactions like suzuki coupling and click reaction, for production of multifaceted deuterated compound (13).

Raman Sharma et al (2012) studied that the pharmacokinetic profile of drugscan be altered by means of deuterium. 


\section{Deuteration as a Tool for Enhancing the Half-Life of Drug}

It was found that the ex-vivoand in-vivo methods suggested that the deuterated carbazeran and zoniporidehave decreased metabolism and it helps in increasing the half life of the drugs.MałgorzataCebo(2014)et al investigated that the exchange in $\mathrm{D}-\mathrm{H}$ in imidazole gets affected by phosphorylation of histidine side chain in peptides. The consequencesreveal that phosphorylation considerablyslow the speed of the DHX reaction. (14)

Maicon Guerra de Miranda develop deuterated compound which is frequently used as biomarkers in petroleum and related sample.(15)The benzopyran deuterated was prepared by Yanmei Zhang, Micky D. Tortorella et al (2014).It is used as novelinhibitor of COX-2.The compound has better pharmacokinetic profile than the previous one. The novel compound showeffective role in various inflmmatory and painful conditions.(16)In 2016 Jinfang Jiang et al studied that the Enzalutamide (ENT) which is responsible for inhibition of the androgen receptor was accepted formanagement of prostate cancer by USFDA.In this the $N$ $\mathrm{CH}_{3}$ moiety were substituted by deuterium. This shows that the drug has good pharmacokinetic profile. (17)

\section{CONCLUSION}

The article concluded that the deuteration is a vitalfactor in enhancing half-life of the drug.The deuterated drugs takes more time to get cleared from the systemic circulation.It helps to increase the residential of drug inside the body and thus helpful in reducing the dosing regimen which can ultimately leads to enhance the dosage regimen.

\section{REFERENCES}

1. Jing Chen, Xiaofang Luo, HuiminQiu, Vienna Mackey, Lichun Sun, Xiaoping OuyangM, Drug discovery and drug marketing with the critical roles of modern administration, Am $\mathrm{J}$ Transl Res 2018;10(12):4302-4312.

2. Benedict, M., Pigford, T.H., and Levi, H.W. ,Nuclear chemical engineering. 2nd ed. McGraw-Hill, New York, 1981:1008.

3. 3.Katz, J.J. 1960. The biology of heavy water. Sci. Am. 203: 106115 .

4. Chauhan P. 2016. Heavy water: alternative applications in biology, medicine and industry.

5. Chauhan P. 2016. Heavy water: alternative applications in biology, medicine and industry .

6. Edward M. Russak,Edward M. Bednarczyk, Impact of Deuterium Substitution on the Pharmacokinetics of Pharmaceuticals, Annals of Pharmacotherapy1-6, 2018.

7. Graham S Timmins,Deuterated drugs; where are we now?,Expert OpinTher Pat. 2014 October ; 24(10): 1067-1075.

8. Scott L. Harbeson, Roger D. Tung, Deuterium Medicinal Chemistry: A New Approach to Drug Discovery and Development,Medchem News No.2 2014:8-22

9. Anwen M. Krause-Heuer, Nageshwar R. Yepuri, Tamim A. Darwishand Peter J. Holden, Mild Conditions for Deuteration of Primary and Secondary Arylamines for the Synthesis of Deuterated Optoelectronic Organic Molecules, Molecules 2014, 19, 1860418617.

10. Robert B. Raffa,Joseph V. Pergolizzi1, Robert Taylor, The First Approved "Deuterated" Drug: A ShortReview of the Concept, Pharmacology \& Pharmacy, 2018, 9, 440-446.

11. Sarah Cargnin1, Marta Serafini\& Tracey Pirali, A primer of deuterium in drug design,FutureMed. Chem. 2019,11(16), 2039-2042

12. Sukhninder Kaur and Monika Gupta,Deuteration as a Tool for Optimization of Metabolic Stability and Toxicity of Drugs,Global journal ofPharmacy \& pharmaceutical Science, 1(4) 2017,1-11

13. Cuibo Liu, Zhongxin Chen, Chenliang Su, Xiaoxu Zhao, QiangGao,Controllabledeuteration of halogenated compounds by photocatalytic D2O splitting, Nature Communications ,(2018) 9:80
14. Raman Sharma, Timothy J. Strelevitz, Hongying Gao, Alan J. Clark, KlaasSchildknegt, R. Scott Obach, Sharon L. Ripp, Douglas K. Spracklin, Larry M. Tremaine, and Alfin D. N. Vaz, Deuterium Isotope Effects on Drug PharmacokineticsSystemDependent Effects of Specific Deuteration with Aldehyde Oxidase Cleared Drugs, Drug Metabolism And Disposition,2017,4(3):625-634

15. Maicon Guerra de Miranda' Andre Luis Mazzei Albert, JariNobrega Cardoso,RosangelaSabbatini Capella Lopes,ClaudioCerqueira Lopes, Straightforward synthesis of 2,2,4,4,5,7,7- $\mathrm{d}_{7}$-cholestane: a new deuterated standard in petroleum analysis, Quim. Nova, 2013, 36(8), 1160-1163.

16. Yanmei Zhang, Micky D. Tortorella,Yican Wang, JianqiLiu,ZhengchaoTu,XiaorongLiu,YangBai,DingshengWen,XinLu ,YongzhiLu,and John J. Talley, Synthesis of Deuterated Benzopyran Derivatives as Selective COX-2 Inhibitors with Improved Pharmacokinetic Properties, ACS Medicinal Chemistry Letters, 2014, $5,1162-1166$

17. JinfangJiang,XuehaiPang,Liang Li , XiaojianDai,XingxingDiao, Xiaoyan Chen, DafangZhong, Yingwei Wang, Yuanwei Chen, Effect of $\mathrm{N}$-methyl deuteration on metabolism and pharmacokinetics of enzalutamide, Drug Design, Development and Therapy 2016:10 2181-2191. 